\title{
Increased Photoconductivity Lifetime in GaAs Nanowires by Controlled $n$-Type and $p$-Type Doping
}

Jessica L. Boland, ${ }^{\dagger}$ Alberto Casadei, ${ }^{\ddagger}$ Gözde Tütüncüoglu, ${ }^{\ddagger}$ Federico Matteini, ${ }^{\ddagger}$ Christopher L. Davies, ${ }^{\dagger}$ Fauzia Jabeen, ${ }^{\ddagger}$ Hannah J. Joyce, ${ }^{\perp}$ Laura M. Herz, ${ }^{\dagger}$ Anna Fontcuberta i Morral, ${ }^{\ddagger}$ and Michael B. Johnston*,

${ }^{\dagger}$ Clarendon Laboratory, Department of Physics, University of Oxford, Parks Road, Oxford, OX1 3PU, United Kingdom

${ }^{\ddagger}$ Laboratory of Semiconductor Materials and ${ }^{\S}$ Laboratory of Quantum Optoelectronics, École Polytechnique Fédérale de Lausanne (EPFL), CH-1015 Lausanne, Switzerland

${ }^{\perp}$ Department of Engineering, University of Cambridge, 9 JJ Thomson Avenue, Cambridge CB3 0FA, United Kingdom

\section{Supporting Information}

ABSTRACT: Controlled doping of GaAs nanowires is crucial for the development of nanowire-based electronic and optoelectronic devices. Here, we present a noncontact method based on timeresolved terahertz photoconductivity for assessing $n$ - and p-type doping efficiency in nanowires. Using this technique, we measure extrinsic electron and hole concentrations in excess of $10^{18} \mathrm{~cm}^{-3}$ for GaAs nanowires with n-type and p-type doped shells. Furthermore, we show that controlled doping can significantly increase the photoconductivity lifetime of GaAs nanowires by over an order of magnitude: from $0.13 \mathrm{~ns}$ in undoped nanowires to 3.8 and $2.5 \mathrm{~ns}$ in $\mathrm{n}$-doped and $\mathrm{p}$-doped nanowires, respectively. Thus, controlled doping can be used to reduce the effects of parasitic surface recombination in optoelectronic nanowire devices, which is promising for nanowire devices, such as solar cells and nanowire lasers.

KEYWORDS: GaAs, n-type doping, p-type doping, terahertz spectroscopy, photoconductivity, mobility, carrier lifetime, surface states, surface recombination, nanowires

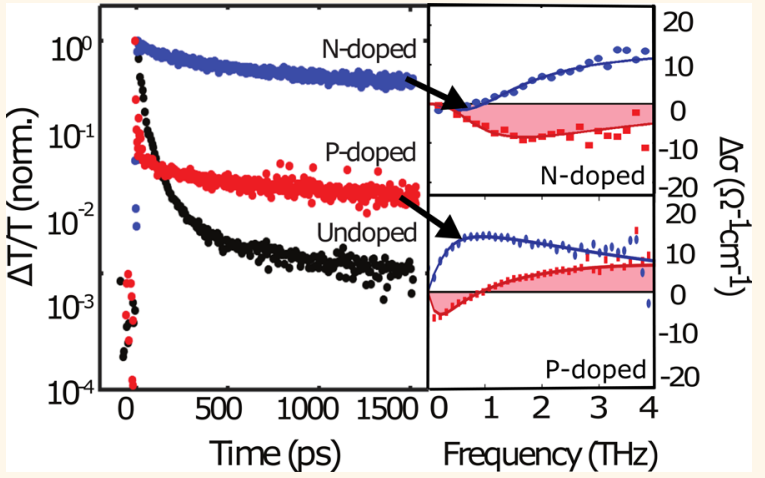

and co-workers in planar GaAs structures, ${ }^{19-21}$ is to exploit engineered band bending via selective doping. Using this technique, they strongly suppressed surface recombination and hence achieved a greater than 3 orders of magnitude increase in radiative efficiencies of bulk GaAs. In this article, we investigate key aspects of the nature of surface recombination in n-doped and p-doped GaAs and show that by exploiting band bending in shell-doped homostructure nanowires, we can almost eliminate surface recombination, leaving radiative (bimolecular) recombination as the primary charge recombination mechanism.

Two important requirements for developing optoelectronic devices from GaAs nanowires is to spatially control the n-type and p-type doping density in nanowires with a sufficiently high range of concentrations and to reduce parasitic SRH

Received: December 1, 2015

Accepted: March 9, 2016 
recombination at nanowire surfaces. Doping in semiconductor nanowires was first investigated almost three decades ago, ${ }^{22}$ yet research in the field has increased rapidly in the past 10 years, following the demonstration of doping in silicon nanowires. ${ }^{23}$ Doping of III-V nanowires, in particular, has been found to be a challenging area of research from both a growth and device perspective. Owing to differences in incorporation paths for lateral and axial growth, dopant incorporation can often lead to inhomogeneous or ineffective doping, compensation, and defects in the crystal structure. ${ }^{24-29}$ Doping of the nanowire core, in particular, has proven difficult due to growth conditions and nanowire morphology. For example, it has proven challenging to obtain an n-type conductivity via Si core doping of $\mathrm{GaAs}$ nanowires due to the amphoretic behavior of $\mathrm{Si}$, as dopants incorporate both on Ga sites as donors and As sites as acceptors. ${ }^{30}$ An n-type conductivity in GaAs nanowires has however been achieved through Te core doping, yet as Te is a high vapor pressure element, its use in high-mobility molecular beam epitaxy (MBE) chambers is not ideal. ${ }^{31,32}$ Alternatively, a shell of doped semiconductor may be grown over a nominally undoped NW core. This "shell doping" technique has allowed many doped semiconductor heterostructures to be realized. $^{33-38}$ More recently, modulation doping has also been achieved in GaAs/AlGaAs core-shell heterostructures. These structures have been shown to possess both high mobilities and long carrier lifetimes, yet relatively low carrier concentrations. ${ }^{39}$ This work aims to continue the investigation into the effects of doping on such GaAs-based nanostructures, by exploring the effects of "shell" doping and different doping types.

Despite advances in doping of GaAs nanowires, detailed studies into their transport properties remain difficult. Accurate measurements of carrier mobility and doping levels face many challenges, due to the quasi one-dimensional geometry of nanowires, which impede conventional Hall techniques, especially in low-diameter nanowires. ${ }^{40}$ Technical challenges in fabricating lateral ohmic contacts, ${ }^{41}$ as well as uncertainty in the gate capacitance term for field-effect mobility measurements, also render such measurements difficult. Noncontact methods for obtaining these key transport parameters, such as Raman spectroscopy ${ }^{42,43}$ and terahertz spectroscopy, ${ }^{39,44-46}$ are therefore of great importance for the progress of the field.

In this article, we examine the ultrafast carrier dynamics of bulk n-type and p-type shell-doped GaAs nanowires and compare the effects of each doping type on carrier mobility and lifetime in the subpicosecond to few nanosecond range. The room-temperature photoconductivity is measured with subpicosecond resolution using optical pump terahertz probe spectroscopy (OPTP). ${ }^{47}$ This noncontact method allows the doping density for both samples, n-type and p-type, to be determined accurately at room temperature without artifacts from electrical contacts. ${ }^{48}$ This is the first time that this technique has been used to accurately extract the carrier concentration in p-doped GaAs nanowires. OPTP measurements confirm that both $\mathrm{Si}$ and $\mathrm{C}$ doping are effective in the GaAs nanowires, with carrier concentrations measured in both the n-type and p-type doped nanowires reaching $(1.3 \pm 0.6) \times$ $10^{18}$ and $(1.4 \pm 0.6) \times 10^{18} \mathrm{~cm}^{-3}$, respectively. Increased impurity scattering in the heavily doped nanowires leads to an order of magnitude drop in electron mobility to $\sim 400 \mathrm{~cm}^{2} \mathrm{~V}^{-1}$ $\mathrm{s}^{-1}$ compared with $\sim 1700 \mathrm{~cm}^{2} \mathrm{~V}^{-1} \mathrm{~s}^{-1}$ for undoped reference nanowires, as expected for heavily doped structures. ${ }^{4,46}$ Significantly, we show that for both n-type and p-type doped GaAs nanowires the photoconductivity lifetime is drastically increased by an order of magnitude in comparison to an undoped reference. The $\mathrm{n}$-doped GaAs nanowires displayed a carrier lifetime of $3.8 \pm 0.1 \mathrm{~ns}$, compared with $0.13 \mathrm{~ns}$ for an undoped reference sample. The p-doped GaAs nanowires exhibited a slightly shorter lifetime of $2.5 \pm 0.02 \mathrm{~ns}$. Together, these results are consistent with a bimolecular recombination constant of $k_{2} \approx 2 \times 10^{-10} \mathrm{~cm}^{3} / \mathrm{s}$ and a near-complete suppression of surface charge recombination pathways for both $\mathrm{n}$ - and p-doped nanowires. We also observed an interesting carrier decay behavior for $\mathrm{p}$-doped nanowires, with a sharp initial decay within 25 ps after photoexcitation. We attribute this decay to rapid electric field assisted trapping of electrons at the surface of the GaAs nanowires after photoexcitation. To our knowledge, this effect has not been seen before in p-type doping studies and provides a novel physical insight into their electronic properties.

\section{RESULTS AND DISCUSSION}

Nanowire Growth. GaAs nanowires were grown on p-type (111) Si substrates using molecular beam epitaxy under optimized conditions to produce a maximum yield of vertical nanowires (details provided in the Supporting Information). ${ }^{49}$ (50 After obtaining the core, conditions were changed for the growth of the doped shell. n-Type doping was obtained by adding silicon during shell growth, ${ }^{51}$ while p-type doping was obtained by adding carbon. ${ }^{52}$ The dimensions of the two samples were similar but not identical: the core and shell were respectively 60 and $45 \mathrm{~nm}$ thick for the n-type nanowires and 70 and $40 \mathrm{~nm}$ thick for the p-type nanowires. The nominal doping concentrations were $2.2 \times 10^{19}$ and $7.6 \times 10^{18} \mathrm{~cm}^{-3}$ respectively for n-type and p-type doping. An undoped reference sample was also produced via MBE under similar growth conditions. The reference sample had the same morphology and geometry as the doped samples, except it did not contain any intentional dopants and is therefore a GaAs nanowire of diameter $115 \mathrm{~nm}$. All samples were transferred to $z$-cut quartz substrates for measurement and aligned with the nanowire axis parallel to the electric field polarization. A direct comparison between these doped samples and an undoped reference then enabled investigations to be conducted into the effects of n-type and p-type doping on the transport properties of the nanowires.

Schrödinger-Poisson Simulation. A self-consistent solution of Schrödinger and Poisson equations ${ }^{53}$ was performed to determine the band edge profile and charge distribution across both n-type and p-type doped nanowires at equilibrium, according to the nominal doping profile. For both doped samples, the donor and acceptor concentrations were set to $2.2 \times 10^{19}$ and $7.8 \times 10^{18} \mathrm{~cm}^{-3}$, respectively, across the entire shell region, based on the nominal doping densities from growth conditions. Figure 1c and e show the calculated electron density profile for the $\mathrm{n}$-doped nanowires. A maximum electron density within the shell of approximately $1.2 \times 10^{18} \mathrm{~cm}^{-3}$ was calculated, dropping to approximately $2 \times 10^{17} \mathrm{~cm}^{-3}$ in the core. The corresponding calculated energy band diagram is depicted in Figure 1a, with the dotted lines representing the core-shell interface. It can be observed from the band diagram that the n-type doping within the shell shifts the position of the Fermi level in the core, causing it to move closer to the conduction band, leading to band bending at the core-shell interface. There is also strong band bending near the surface of the nanowire, resulting from Fermi-level pinning at midgap surface states, ${ }^{54}$ which leads to an electron depletion region at 


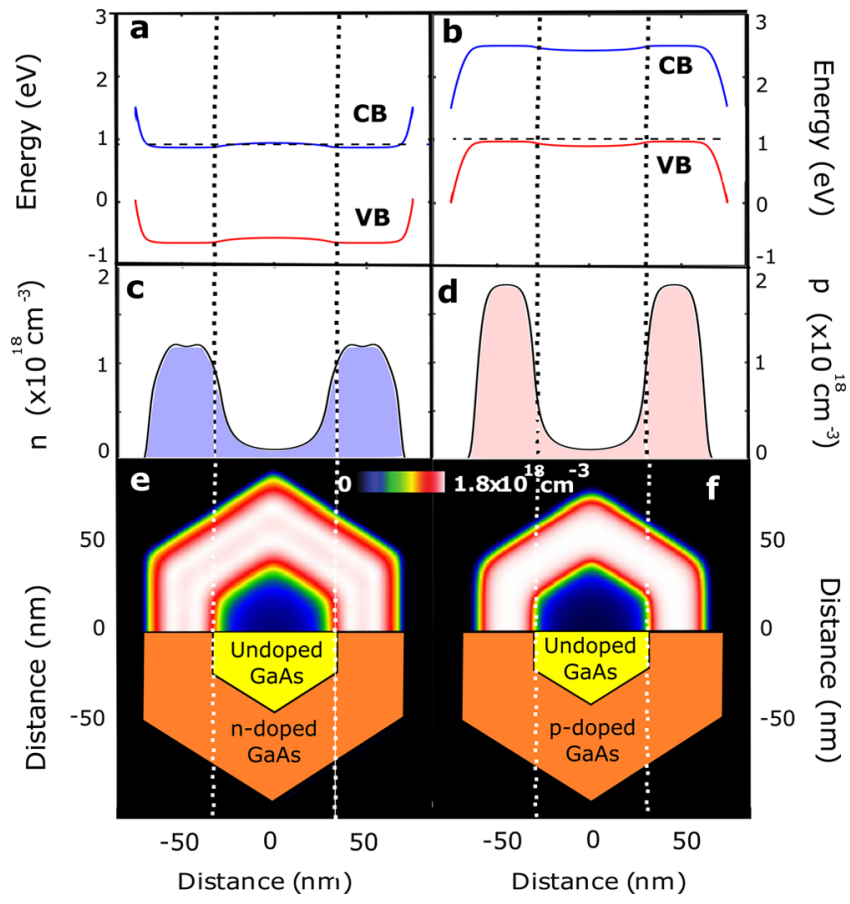

Figure 1. Top: Energy band diagrams for both n-doped (a) and pdoped (b) nanowires. The conduction band edge is shown in blue, the valence band edge in red, and the Fermi level in dashed black. The distance plotted represents the distance from the center of the nanowire, and the dashed line marks the core-shell interface. Second Row: One-dimensional plot of electron density (c) and hole density profile (d) plotted as a function of distance across the nanowire. The cross-section for which the density profiles are plotted is represented as a black line on density profiles. Third Row: Nextnano ${ }^{53}$ simulations of the electron density (e) and hole density profiles (f) for the $n$-doped and p-doped nanowires, respectively, assuming nominal doping densities of $2.2 \times 10^{19}$ and $7.6 \times 10^{18} \mathrm{~cm}^{-3}$ respectively for $\mathrm{n}$-type and p-type doping. A schematic diagram of the nanowire structure is superimposed on the lower half of the images for both samples.

the surface. For the p-doped nanowires, the calculated hole density is plotted in Figure $1 \mathrm{~d}$ and $\mathrm{f}$, again showing a maximum hole density of approximately $1.8 \times 10^{18} \mathrm{~cm}^{-3}$ within the shell, where the acceptor ions are situated, dropping to $2 \times 10^{17} \mathrm{~cm}^{-3}$ in the center of the core. The corresponding energy band diagram is shown in Figure $1 \mathrm{~b}$, with the dotted lines again representing the core-shell interface. The p-type doping within the shell has the opposite sign on the band structure compared to the $\mathrm{n}$-doped sample, with the Fermi level in the core shifting closer to the valence band, leading to band bending at the core-shell interface. The Fermi level is again pinned at the surface, ${ }^{54}$ causing band bending at the surface of the nanowire. Comparing the density profiles for both samples, the hole density within the core for the p-doped sample is slightly lower than the electron density within the core for the n-doped sample. This is due to the larger core diameter and thinner doped shell for the p-doped sample. However, for both doped samples, it can be seen that Fermi level pinning and diffusion at the core-shell interface lead to a redistribution of extrinsic charge carriers toward the core-shell interface and into the core region.

Photoconductivity Measurements. The carrier dynamics in the frequency domain and time-resolved room-temperature photoconductivity were measured for both the n-type and $\mathrm{p}$ - type doped GaAs nanowires and the undoped reference using the OPTP setup described in the Methods section. ${ }^{14,39}$ Scanning electron microscope (SEM) images in Figure 2

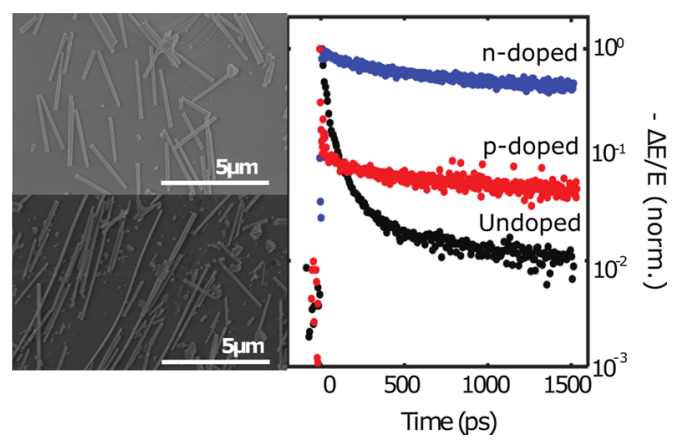

Figure 2. Left: SEM images for the n-type (top) and p-type (bottom) GaAs nanowires as transferred onto quartz substrates. Further SEM images of nanowires as grown can be found in Supporting Information Figure S1. Right: Comparison of the decay of normalized photoconductivity for bulk n-type (blue) and bulk ptype (red) doped nanowires with an undoped nanowire reference sample (black). The excitation photon energy, fluence, and pulse duration were $1.55 \mathrm{eV}, 25.5 \mu \mathrm{J} \mathrm{cm}^{-2}$, and $35 \mathrm{fs}$, respectively. All measurements were performed at room temperature.

depict representative nanowires on quartz substrates, showing that a high density of nanowires were transferred. The nanowires were photoexcited with a near-infrared laser at a wavelength of $800 \mathrm{~nm}\left(E_{\text {photon }}=1.55 \mathrm{eV}\right)$ with pulses of $35 \mathrm{fs}$ duration. Photoexcitation at this wavelength allows electronhole pairs to be generated across the whole nanowire uniformly. The nanowires were photoexcited at fluences between 5 and 77 $\mu \mathrm{J} \mathrm{cm}{ }^{-2}$. This photoexcitation induces a change, $\Delta E$, in the electric field transmission of the terahertz probe pulse, $E$. The ratio of this change to the terahertz probe transmission, $|\Delta E / E|$, is a function of the photoinduced conductivity $\Delta \sigma$ of the nanowires and thereby the change in free carrier concentration $^{55}$ (see Supporting Information). This photoinduced conductivity can then be measured as a function of time after photoexcitation and as a function of frequency to extract the doping densities, carrier mobilities, and carrier lifetimes. Thus, OPTP spectroscopy may be thought of as an electrical transport measurement technique with very high temporal resolution since it probes conductivity dynamics. The terahertz conductivity signal is dominated by the charge species that have the highest product of carrier density and mobility (as is also the case in Hall measurements). For example, electrons in GaAs have roughly an order of magnitude higher mobility $\mu_{\mathrm{e}}$ than heavy holes $\mu_{\mathrm{hh}}$. Thus, in order for the terahertz conductivity, $\sigma_{\mathrm{THz}}=n e \mu_{\mathrm{e}}+p e \mu_{\mathrm{hh}}$ to be dominated by holes, the hole density $p$ must be at least an order of magnitude greater than the electron density $n$. In contrast to $\mathrm{THz}$ conductivity measurements, time-resolved photoluminescence spectroscopy probes the dynamics of minority charge carriers.

Photoconductivity Dynamics of Doped Nanowires. Figure 2 shows the change in nanowire photoconductivity as a function of time after photoexcitation for the n-type and p-type doped samples in comparison with the undoped reference sample. The effect of photoexcitation fluence on the photoconductivity dynamics in the frequency domain for all samples is shown in Figure 3, with measurements taken at fluences of 5, 13 , and $26 \mu \mathrm{J} \mathrm{cm}^{-2}$. For both doping types, the photo- 

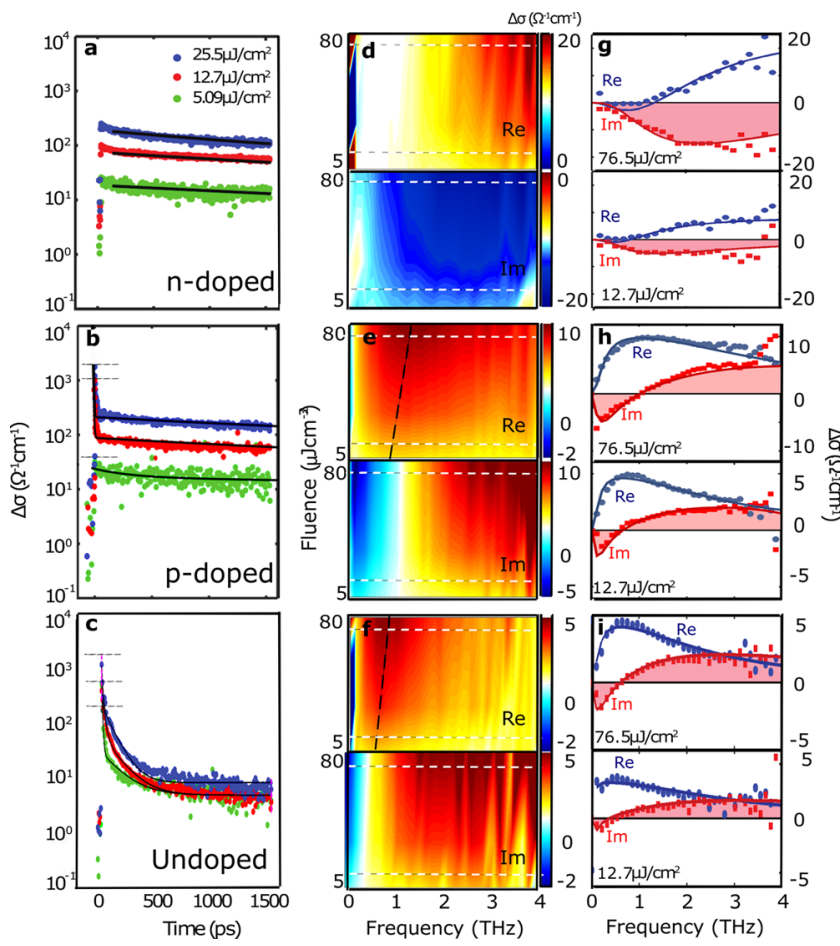

Figure 3. $(a-c)$ Photoinduced change of electrical conductivity in n-type and p-type doped and undoped GaAs nanowires as a function of time after photoexcitation by 35 fs pulses of $1.55 \mathrm{eV}$ photons at fluences of $5,12.7$, and $25.5 \mu \mathrm{J} \mathrm{cm}$. The symbols represent the measured data, the solid black lines the fitted carrier rate equations, and the horizontal lines the start of each fluence response. $(d-f)$ Color maps of the real and imaginary parts of timeresolved conductivity as a function of frequency and of fluence for n-doped (top), p-doped (middle), and undoped (bottom) nanowires. Dashed black lines represent the position of the resonant frequency. $(g-i)$ Sample spectra for the time-resolved conductivity of photoexcited electrons as a function of frequency for the n-type (top), p-type (middle), and the undoped (bottom) nanowires taken at a time of 5 ps after photoexcitation with fluences of 12.7 and $76.5 \mu \mathrm{J} \mathrm{cm}^{-2}$. These fluences correspond to the white dashed lines on the color maps. The symbols represent the measured data and the solid lines the fitted plasmon responses. The real (blue) and imaginary (red) components of the conductivity are plotted. All measurements were performed at room temperature.

conductivity clearly shows a rapid rise when photoexcited, followed by a slower decay.

Nonequilibrium charge recombination in an intrinsic (undoped) semiconductor in the absence of electron-hole correlations may be described in terms of a rate equation:

$$
\frac{\mathrm{d} n}{\mathrm{~d} t}=-k_{1} n-k_{2} n^{2}-k_{3} n^{3}
$$

where $n$ is the intrinsic electron concentration (which is equal to the intrinsic hole cencentration $p$ ). The rate constant $k_{1}$ describes recombination via defects and surface states, $k_{2}$ is the radiative (bimolecular) rate constant, and $k_{3}$ is the constant describing Auger processes. ${ }^{39,56}$ For an n-doped semiconductor with extrinsic electron density $n_{0}$ the equation becomes

$$
\begin{aligned}
\frac{\mathrm{d} n}{\mathrm{~d} t} & =-k_{1} n-k_{2}\left(n+n_{0}\right) n-k_{3} n^{3} \\
& =-\left(k_{1}+k_{2} n_{0}\right) n-k_{2} n^{2}-k_{3} n^{3}
\end{aligned}
$$

Replacing $n_{0}$ with the extrinsic hole density $p_{0}$ in this expression gives the corresponding expression for a p-doped semiconductor.

The fluence-dependent photoconductivity data shown in Figure 3 a were globally fitted using eq 2 . Second- and thirdorder recombination processes were found to be negligible, and a monoexponential recombination lifetime $\tau_{n}=1 /\left(k_{1}+k_{2} n_{0}\right)=$ $3.8 \pm 0.1 \mathrm{~ns}$ was extracted. Note, eq 2 is not valid at early times when $k_{1}$ varies owing to dynamic band bending (as described later); thus fitting was performed for times greater than 50 ps after photoexcitation (as indicated by the solid lines in Figure $3 a-c)$.

For the p-doped GaAs nanowires (Figure 3b), there is an initial sharp decay within the first 25 ps after photoexcitation, with the free carrier concentration rapidly decreasing by an order of magnitude. This sharp initial decay has not been seen before with p-type doping and can be explained in terms of band bending and electron trapping at surface states, as described in detail later. Upon direct comparison of the decay behavior after 200 ps (Figure 2), both doped samples exhibit significantly longer carrier lifetimes than the undoped reference. The decay for the undoped nanowires was fitted with a biexponential to account for the sharp decay due to trapping, which had a lifetime of $6.9 \pm 0.1 \mathrm{ps}$, and the slower monoexponential decay of excess electrons and holes, which exhibited a carrier lifetime of $162 \pm 4$ ps. This suggests that the increase in photoconductivity lifetime for both doped samples is a direct effect of doping the nanowires. On photoexcitation, the equilibrium band bending, illustrated in Figure 1a and b, is flattened as a result of screening (as shown in Supporting Information Figure S2a and b). However, as charge carriers begin to recombine, band bending and thus surface electric fields reappear. The surface depletion layer for the n-type nanowires helps to keep the electron population away from the trap-rich surface of the nanowire, significantly reducing surface recombination of electrons. In contrast to the n-type nanowires, the opposite direction of band bending in the p-type nanowires helps drive photoinjected minority electrons toward the defective surface, leading to the fast initial decay in photoconductivity. However, it also confines holes away from the surface, leading to a long hole-dominated photoconductivity after 200 ps. Therefore, the photoconductivity decay for the pdoped GaAs nanowires can be fitted with a biexponential, which accounts for the fast initial decay due to electron trapping and the slower monoexponential decay of holes within the nanowire. The fast initial decay then exhibits a lifetime of $3.3 \pm 0.2 \mathrm{ps}$ and the slower hole-dominated photoconductivity decay a lifetime of $\tau_{\mathrm{p}}=1 /\left(k_{1}+k_{2} p_{0}\right)=2.5 \pm 0.02 \mathrm{~ns}$. This long carrier lifetime coincides with previous time-resolved thermoelectric transport measurements of p-type GaAs nanowires, suggesting it is a direct effect of the inclusion of dopants. ${ }^{38}$ Thus, both n-type and p-type doped nanowires display longer photoconductivity lifetimes in comparison to undoped reference nanowires, highlighting doping as an alternative technique to surface passivation for tailoring photoconductivity lifetimes for nanowire-based devices.

Interpretation of Nanowire Photoconductivity Spectra. To further investigate these recombination mechanisms and carrier-carrier scattering within these doped nanowires, photoconductivity spectra were obtained for all samples. Figure 3 shows the photoconductivity spectra for the n-doped, $p$ doped, and undoped GaAs nanowires, respectively. Spectra were taken at 5 ps after photoexcitation at excitation fluences of 
$13,26,51$, and $77 \mu \mathrm{J} \mathrm{cm}^{-2}$. A clear Lorentzian resonance in the photoconductivity at terahertz frequencies was seen for all samples. For the n-doped GaAs nanowires (Figure 3d and $\mathrm{g}$ ), the plasma frequency is shifted beyond $4 \mathrm{THz}$ owing to the addition of a high carrier concentration of extrinsic dopants, which shifts the peak in the Lorentzian response beyond the bandwidth of our OPTP system. However, the plasma frequencies for the nominally undoped (Figure $3 f$ and $i$ ) and p-doped (Figure $3 e$ and h) GaAs nanowires fall within the bandwidth of the system and can be seen clearly in the photoconductivity spectra. The p-type doping also causes the plasma frequency to shift and increase in frequency in comparison to the undoped reference. However, the shift in frequency is less drastic due to the large hole effective mass. The dashed black lines in the spectral color maps in Figure 3 clearly show the resonant frequency for both the p-doped and undoped GaAs nanowires. It can be observed that the resonant frequency red-shifts with decreasing excitation fluence. This reduction of resonant frequency with decreasing electron density is a key feature of localized surface plasmon (LSP) modes, confirming the surface plasmon response for the photoconductivity of these nanowires. ${ }^{57}$

The complex photoconductivity of a free carrier plasma with a surface plasmon resonance can be described by the following equation:

$$
\Delta \sigma\left(n, m^{*}, \gamma\right)=\frac{\mathrm{i} n e^{2} \omega}{m^{*}\left(\omega^{2}-\omega_{0}^{2}+\mathrm{i} \omega \gamma\right)}
$$

where $n$ is the carrier density, $e$ is the electronic charge, $m^{*}$ is the effective mass of the charge carrier, and $\gamma$ is the momentum scattering rate. $\omega_{0}$ is the surface plasmon resonance frequency given by

$$
\omega_{0}\left(n, m^{*}\right)=\sqrt{\frac{f n e^{2}}{m^{*} \epsilon_{\mathrm{r}} \epsilon_{0}}}
$$

where $\epsilon_{\mathrm{r}}$ is the dielectric constant of GaAs nanowires at terahertz frequencies, $\epsilon_{0}$ is the permittivity of free space, and $f$ is a constant that depends on the nanowire geometry and surrounding dielectric medium. ${ }^{44}$ In order to account for the doping within the samples, the photoconductivity expression must be modified, as there is a charge carrier density present at equilibrium without photoexcitation that produces its own plasmon response. ${ }^{39,58}$ As there is a significant equilibrium hole carrier concentration $p_{0}$ in the case of p-type doping, the hole photoconductivity must also be considered before and after photoexcitation. In previous studies, ${ }^{45,59,60}$ the contribution of holes to the conductivity has been neglected owing to their high effective mass. The hole conductivity should be negligible for both the $\mathrm{n}$-doped and undoped reference sample, yet will have a significant effect on the photoconductivity spectral response for the p-doped sample. When both intrinsic and extrinsic carriers and hole conductivity are considered, the complex photoconductivity becomes

$$
\begin{aligned}
\Delta \sigma=\left(\sigma_{\text {electron,excited }}-\right. & \left.\sigma_{\text {electron,unexcited }}\right)+\left(\sigma_{\text {hole,excited }}-\sigma_{\text {hole,unexcited }}\right) \\
\Delta \sigma= & \frac{\mathrm{i} e^{2} \omega}{m_{\mathrm{e}}^{*}}\left[\frac{n+n_{0}}{\omega^{2}-\left\{\omega_{0}\left(n+n_{0}, m_{\mathrm{e}}^{*}\right)\right\}^{2}+i \omega \gamma_{\mathrm{e}}}\right. \\
& \left.-\frac{n_{0}}{\omega^{2}-\left\{\omega_{0}\left(n_{0}, m_{\mathrm{e}}^{*}\right)\right\}^{2}+\mathrm{i} \omega \gamma_{\mathrm{e}}}\right] \\
+ & \frac{\mathrm{ie} e^{2} \omega}{m_{\mathrm{h}}^{*}}\left[\frac{p+p_{0}}{\omega^{2}-\left\{\omega_{0}\left(p+p_{0}, m_{\mathrm{h}}^{*}\right)\right\}^{2}+\mathrm{i} \omega \gamma_{\mathrm{h}}}\right. \\
& \left.-\frac{p_{0}}{\omega^{2}-\left\{\omega_{0}\left(p_{0}, m_{\mathrm{h}}^{*}\right)\right\}^{2}+\mathrm{i} \omega \gamma_{\mathrm{h}}}\right]
\end{aligned}
$$

where $n$ is the photoexcited electron density, $p$ is the photoexcited hole density, and we set $n=p . n_{0}$ is the donated electron density and $p_{0}$ is the donated hole density. $m_{\mathrm{e}}^{*}$ and $m_{\mathrm{h}}^{*}$ are the electron effective mass and hole effective mass, respectively. $\gamma_{\mathrm{e}}$ is the electron scattering rate and $\gamma_{\mathrm{h}}$ is the hole scattering rate. The photoconductivity for doped samples is thus a subtraction of Lorentzian responses: due to a combination of photoexcited electrons and extrinsic charge carriers after photoexcitation; and solely due to extrinsic carriers before photoexcitation.

For each sample, a global fitting routine was applied to all the photoconductivity spectra at various photoexcitation fluences with $f$ fixed to $0.25 .^{39} n_{\mathrm{d}}$ and $n_{\mathrm{a}}$ were both set to global parameters, remaining constant for all spectra. $n_{\mathrm{p}}, \gamma_{\mathrm{e}}$, and $\gamma_{\mathrm{h}}$ were allowed to vary as local parameters for each spectrum, as the photoexcited electron density and scattering rates vary with photoexcitation fluence. Bulk values for GaAs of $0.063 m_{e}$, $0.51 m_{\mathrm{e}}$, and 12.95 were used for $m_{\mathrm{e}}^{*}, m_{\mathrm{h}}^{*}$, and $\epsilon_{\mathrm{r}}$, respectively. ${ }^{61}$ The solid lines in Figure 3 represent the fits for eq 5 with the measured photoconductivity spectra, showing excellent agreement for all samples. From these fits, the carrier mobilities and doping levels were extracted.

Electron and Hole Mobilities of Doped Nanowires. In general, electron and hole mobilities differ due to the difference in effective masses for electrons and holes and the scattering processes affecting each carrier type. The electron mobility for the n-type doped GaAs nanowires was found to be $460 \pm 63$ $\mathrm{cm}^{2} \mathrm{~V}^{-1} \mathrm{~s}^{-1}$ and for the p-type doped GaAs nanowires to be $380 \pm 57 \mathrm{~cm}^{2} \mathrm{~V}^{-1} \mathrm{~s}^{-1}$ at the highest fluence. These mobilities are significantly reduced due to impurity scattering in comparison to the undoped reference, which possessed an electron mobility of $1700 \pm 270 \mathrm{~cm}^{2} \mathrm{~V}^{-1} \mathrm{~s}^{-1}$. The hole mobilities could not be extracted for the n-doped sample, owing to the much higher electron conductivity dominating the measured signal. The hole mobility for the p-doped sample was found to be $48 \pm 12 \mathrm{~cm}^{2} \mathrm{~V}^{-1} \mathrm{~s}^{-1}$ at the highest fluence at a time of 250 ps after photoexcitation, where the photoconductivity is hole-dominated. This hole mobility for the p-doped sample is low and similar to values obtained by other studies. ${ }^{43}$ This reduction in carrier mobility is a key feature of bulk doping in semiconductor nanowires and is attributed to scattering between charge carriers and impurities. ${ }^{43}$ However, this phenomenon has been shown to be minimized via modulation doping in planar heterostructures ${ }^{62}$ and also recently in coreshell nanowires. ${ }^{39}$ Doping levels for both the bulk n-type and $\mathrm{p}$ type GaAs were also extracted from the global fits shown in Figure 3. The n-doped sample was found to have an average doping density of $n_{0}=(1.3 \pm 0.6) \times 10^{18} \mathrm{~cm}^{-3}$ and the $\mathrm{p}$ doped sample to have a doping density of $p_{0}=(1.4 \pm 0.6) \times$ $10^{18} \mathrm{~cm}^{-3}$. These doping concentrations agree with simulations 
and are very high, showing that bulk doping within the shell of GaAs nanowires can successfully achieve high doping levels, which is promising for future nanowire-based electronics.

Radiative versus Nonradiative Recombination. Having established $n_{0}$ and $p_{0}$ and extracted $\tau_{\mathrm{n}}=1 /\left(k_{1}+k_{2} n_{0}\right)=3.8 \pm$ $0.1 \mathrm{~ns}$ and $\tau_{\mathrm{p}}=1 /\left(k_{1}+k_{2} p_{0}\right)=2.5 \pm 0.02 \mathrm{~ns}$ for the $\mathrm{n}$-doped and $\mathrm{p}$-doped nanowires from global fits to the data in Figure $3 \mathrm{a}$ and $\mathrm{b}$, the radiative (bimolecular) recombination constant for these samples may be calculated. In this case, $k_{1} \approx 0$ and the radiative recombination constant is $k_{2}=(2.0 \pm 0.9) \times 10^{-10}$ $\mathrm{cm}^{3} \mathrm{~s}^{-1}$ and $k_{2}=(2.8 \pm 1.2) \times 10^{-10} \mathrm{~cm}^{3} \mathrm{~s}^{-1}$ for the n-type and p-type nanowires. Both these values agree with the established value of $k_{2} \approx 2 \times 10^{-10} \mathrm{~cm}^{3} \mathrm{~s}^{-1},{ }^{10,38,56}$ validating the choice of $k_{1} \approx 0$. Therefore, these results indicate that nonradiative recombination routes, such as recombination via defects and surface states, are strongly suppressed in shell-doped nanowires. This hypothesis is also supported by a marked increase in photoluminescence (PL) efficiency as a result of shell doping. For example, while no PL could be detected from the undoped reference nanowires at room temperature, strong PL emission was observed from the p-type nanowires (see Figure S4 of the Supporting Information).

Recombination Dynamics in p-Doped Nanowires. To further investigate the effects of doping, p-type doping in particular, on the carrier dynamics of the GaAs nanowires, photoconductivity spectra were also taken at different times after photoexcitation. For the n-type doped and undoped GaAs nanowires, a Lorentzian response was displayed for all times after photoexcitation, as shown in the Supporting Information. The plasma frequency decreased with increasing time after photoexcitation, as expected for LSP modes. However, for the p-type doped GaAs nanowires, a clear Lorentzian response was no longer observed for times greater than 25 ps after photoexcitation. Figure $4 \mathrm{a}$ shows the normalized photoconductivity for the p-doped sample plotted as a function of time after photoexcitation for an excitation fluence of $26 \mu \mathrm{J}$ $\mathrm{cm}^{-2}$. Photoconductivity spectra for times of 25 and $1000 \mathrm{ps}$ after photoexcitation are plotted in Figure $4 b$ and c, with arrows marking the point on the decay curve to which they correspond. At 25 ps after photoexcitation, the photoconductivity is decaying rapidly and a Lorentzian response is seen. At this time, the equilibrium band bending is flattened due to screening and the conductivity is dominated by electrons in the conduction band (see Supporting Information Figure S3 for a diagram of the simulated band bending). In contrast, by $1000 \mathrm{ps}$, the photoconductivity is decaying at a slower rate and a Lorentzian response is no longer observed. The imaginary part of the photoconductivity is close to zero, and the real part of the photoconductivity is a constant positive value for frequencies above $100 \mathrm{GHz}$. At this time, the majority of electrons have moved to the nanowire surface as a result of band bending and either recombined or fallen into trap states, leaving holes as the majority photoexcited free carriers for this conductivity response. The physical interpretation is that holes no longer experience localization due to the nanowire on account of their shorter mean free path, which was calculated to be much smaller than the dimensions of the nanowire at $8.7 \mathrm{~nm}$ (see Supporting Information). Therefore, a Drude conductivity response with a short scattering time is observed. This suggests that the conductivity at $1000 \mathrm{ps}$ is mainly a result of the remnant holes within the nanowire.
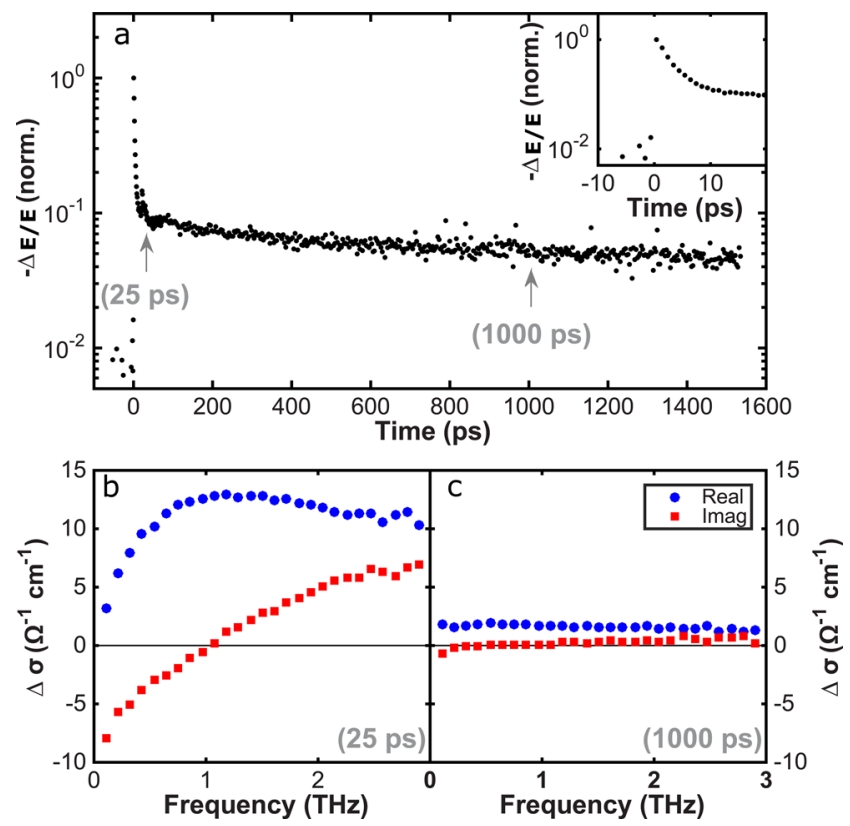

Figure 4. (a) Decay of the normalized photoconductivity for bulk ptype doped GaAs nanowires as a function of time after photoexcitation. Time-resolved conductivity spectra extracted at delay times of (b) 25 ps and (c) 1000 ps after photoexcitation. Spectra correspond to points on the photoconductivity decay curve marked by arrows. The nanowires were photoexcited by $35 \mathrm{fs}$ pulses of $1.55 \mathrm{eV}$ photons at a fluence of $25.5 \mu \mathrm{J} \mathrm{cm}$.

\section{CONCLUSIONS}

In conclusion, we have presented the first noncontact terahertz spectroscopy measurements of GaAs nanowires with bulk ntype and p-type shell doping. We show that the OPTP technique enables carrier lifetimes, carrier mobilities, and doping concentrations to be accurately determined, as well as recombination mechanisms investigated in detail using timedependent conductivity spectral data. We have demonstrated that successful bulk n-type and p-type doping within the nanowire shell can be achieved with doping densities of $(1.3 \pm$ $0.6) \times 10^{18} \mathrm{~cm}^{-3}$ and $(1.4 \pm 0.6) \times 10^{18} \mathrm{~cm}^{-3}$, respectively. Such high doping concentrations are essential for future nanowire-based devices. From photoconductivity spectra, we have extracted electron mobilities of $460 \pm 63$ and $380 \pm 57$ $\mathrm{cm}^{2} \mathrm{~V}^{-1} \mathrm{~s}^{-1}$ for the $\mathrm{n}$-doped and p-doped samples, respectively. A hole mobility of $48 \pm 12 \mathrm{~cm}^{2} \mathrm{~V}^{-1} \mathrm{~s}^{-1}$ was also extracted for the p-doped sample. These carrier mobilities are low in comparison to an undoped reference, yet could be improved through modulation doping. ${ }^{33-37,39,42}$ More importantly, we have demonstrated an increase in photoconductivity lifetime in comparison to an undoped reference. The photoconductivity lifetime was found to be $3.8 \pm 0.1 \mathrm{~ns}$ for n-type doping and 2.5 $\pm 0.02 \mathrm{~ns}$ for $\mathrm{p}$-type doping. These results demonstrate a strong enhancement of radiative over nonradiative surface and defect mediated recombination, which is promising for the use of doped nanowires in optoelectronic devices, such as lasers and solar cells. Furthermore, we have shown a novel effect of p-type doping in GaAs nanowires, with an initial sharp decay seen within 25 ps after photoexcitation. Through analysis of timedependent conductivity spectra, we have attributed this fast decay to rapid trapping of electrons at the nanowire surface, assisted by doping related band bending. This is an interesting insight into the effects of doping on the carrier dynamics in $\mathrm{p}$ - 
doped nanowires and highlights terahertz spectroscopy as a reliable technique for characterization of doped semiconductor nanowires.

\section{METHODS}

Nanowire Growth. GaAs nanowires used in the study were grown in a DCA P600 molecular beam epitaxy machine. The substrates were (111) silicon wafers with native oxide. Maximum yield of the vertical nanowires was obtained by native oxide engineering and optimization of V/III, in order to suppress twinning. ${ }^{50}$ The "core" part of the nanowire was grown via a self-catalyzed growth technique, using gallium droplets. Nanowire growth conditions were altered slightly depending on the required specifications. For the undoped sample, the GaAs growth rate was $1 \AA / \mathrm{s}$ and the arsenic flux was $2.5 \times 10^{-6}$ Torr. Growth is continued at $640{ }^{\circ} \mathrm{C}$ for $60 \mathrm{~min}$ with $7 \mathrm{rpm}$ rotation, resulting in nanowires with approximately $6 \mu \mathrm{m}$ length and $110 \mathrm{~nm}$ $( \pm 10 \mathrm{~nm})$ diameter. A tilted view and cross-section of the nanowire forest is shown in Supporting Information Figure S1.

For doped samples, the Ga flux was reduced to $0.25 \AA / \mathrm{s}$, which resulted in a reduction of the diameter of the core. ${ }^{50}$ Following the core growth, the gallium droplet was consumed by only supplying arsenic flux, and the growth conditions were then changed toward the growth conditions of (110) GaAs epitaxial growth, which requires a lower temperature and higher V/III ratio. For n-doping, the current of the silicon cell was ramped-up to $45 \mathrm{~A}$, and for p-doping the current of the carbon cell was ramped-up to $48 \mathrm{~A}$. According to the Hall measurements performed on doped (100) GaAs wafers, these values correspond to $2.2 \times 10^{19} \mathrm{~cm}^{-3}$ for $\mathrm{n}$-doping and $7.6 \times 10^{18} \mathrm{~cm}^{-3}$ for $\mathrm{p}$ doping.

Preparation of Samples for Photoconductivity and Photoluminescence Measurements. All samples were transferred to 2 $\mathrm{mm}$ thick $z$-cut quartz substrates. They were all rubbed in the same direction, leading to nanowires lying flat on the surface of the quartz substrate with a preferential alignment direction (see Figure 2). In all experiments the samples were aligned such that the long axis of the majority of nanowires was parallel to the electric field direction of both the pump laser pulse and the $\mathrm{THz}$ transient. Ensembles of nanowires were measured with an areal density of approximately 1900 and 2650 nanowires per $\mathrm{mm}^{2}$, for $\mathrm{n}$-type and $\mathrm{p}$-type samples respectively. Due to the inhomogeneous averaging of the nanowire size distribution, all the mobility values represent lower bounds.

Terahertz Photoconductivity Measurements. Time and spectrally resolved photoconductivity measurements were performed using the technique of optical pump terahertz probe spectroscopy. A regenerative Ti:sapphire laser amplifier (Spectra Physics Spitfire Pro) was used to generate $35 \mathrm{fs}$ pulses centered at $800 \mathrm{~nm}$ at a repetition rate of $5 \mathrm{kHz}$ with an average power of $4 \mathrm{~W}$. Each pulse was separated into three different paths: $590 \mu \mathrm{J} /$ pulse as an optical pump to photoexcite the sample; $200 \mu \mathrm{J} /$ pulse to generate the $\mathrm{THz}$ probe via optical rectification in a $2 \mathrm{~mm} \mathrm{GaP}$ crystal; and $1.6 \mu \mathrm{J} /$ pulse as a gate beam for electro-optical detection of the transmitted $\mathrm{THz}$ pulse via a $200 \mu \mathrm{m} \mathrm{GaP}$ crystal. In order to obtain a range of sample photoexcitation fluences between 5 and $26 \mu \mathrm{J} \mathrm{cm}^{-2}$, the optical pump beam was attenuated by neutral density filters. At the sample, the full width at half-maximum (fwhm) for the optical pump beam is $10 \mathrm{~mm}$ and for the $\mathrm{THz}$ probe is $1 \mathrm{~mm}$, so that the $\mathrm{THz}$ probe measures an area of homogeneous photoexcited carrier density. The $\mathrm{THz}$ electric field, $E$, was measured using a balanced photodiode circuit and a lock-in amplifier referenced to a chopper at $2.5 \mathrm{kHz}$ in the $\mathrm{THz}$ generation beam. The optical pump-induced change in the $\mathrm{THz}$ electric field, $\Delta E$, was measured using a second lock-in amplifier referenced to a chopper at $125 \mathrm{~Hz}$ in the optical pump beam. By varying the time delays between all three beams, a $2 \mathrm{D}$ map of the $\mathrm{THz}$ spectral response as a function of time after photoexcitation can then be measured. By also varying the power of the optical pump beam, a $\mathrm{THz}$ spectra response as a function of photoexcitation fluence can also be obtained. All measurements were taken at room temperature with the $\mathrm{THz}$ beam under vacuum to avoid any absorption of $\mathrm{THz}$ radiation by atmospheric water vapor. Details of the analysis of the data are provided in the Supporting Information.

Ensemble Photoluminescence Measurements. Time-integrated photoluminescence measurements were performed on the ensembles of nanowires using a system that has been described in detail previously. ${ }^{63}$ Briefly, samples were excited at a wavelength of 780 $\mathrm{nm}$, with $40 \mathrm{~mW}$ average power on a spot of area $\sim 0.2 \mathrm{~mm}^{2}$ using a mode-locked Ti:sapphire oscillator with a pulse duration of $100 \mathrm{fs}$ and $80 \mathrm{MHz}$ repetition rate. The photoluminescence was passed though a $790 \mathrm{~nm}$ long-pass filter and a $160 \mathrm{~mm}$ spectrometer (grating 300 grooves $/ \mathrm{mm}$ ) onto a silicon CCD camera.

Schrödinger-Poisson Simulation. The band edge profiles and charge carrier distributions in the nanowire homostructures were simulated using a commercial Schrödinger-Poisson current solver (netxnano $\mathrm{GmbH}$ ). ${ }^{53}$ Further details of the simulation parameters can be found in the Supporting Information.

\section{ASSOCIATED CONTENT}

\section{S Supporting Information}

The Supporting Information is available free of charge on the ACS Publications website at DOI: 10.1021/acsnano.5b07579.

Scanning electron microscopy images of as-grown samples; Schrödinger-Poisson simulation results for photoexcited nanowires and recombination; photoconductivity dynamics data for undoped nanowires as a function photoexcitation fluence; time-dependent photoconductivity spectra for $\mathrm{n}$-doped and undoped nanowires; details of data analysis (calculations for converting terahertz transmission data to photoconductivity, descriptions of rate equations used for modeling of timeresolved conductivity data $(\mathrm{PDF})$

\section{AUTHOR INFORMATION}

\section{Corresponding Author}

*E-mail: michael.johnston@physics.ox.ac.uk.

\section{Notes}

The authors declare no competing financial interest.

\section{ACKNOWLEDGMENTS}

The authors thank the EPSRC (U.K.) for financial support via project EP/M017095/1. The Swiss National Science Foundation via projects 137648 and 156081, the NCCR-QSIT, and the NanoTera Program though the Synergy Project are greatly acknowledged.

\section{REFERENCES}

(1) Li, Y.; Qian, F.; Xiang, J.; Lieber, C. M. Nanowire Electronic And Optoelectronic Devices. Mater. Today 2006, 9, 18.

(2) Gradevcak, S.; Qian, F.; Li, Y.; Park, H.; Lieber, C. M. GaN Nanowire Lasers with Low Lasing Thresholds. Appl. Phys. Lett. 2005, $87,173111$.

(3) Duan, X. F.; Huang, Y.; Agarwal, R; Lieber, C. M. SingleNanowire Electrically Driven Lasers. Nature 2003, 421, 241.

(4) Saxena, D.; Mokkapati, S.; Parkinson, P.; Jiang, N.; Gao, Q.; Tan, H. H.; Jagadish, C. Optically Pumped Room Temperature GaAs Nanowire Lasers. Nat. Photonics 2013, 7, 963-968.

(5) Duan, X. F.; Huang, Y.; Cui, Y.; Wang, J. F.; Lieber, C. M. Indium Phosphide Nanowires as Building Blocks for Nanoscale Electronic and Optoelectronic Devices. Nature 2001, 409, 66-69.

(6) Minot, E. D.; Kelkensberg, F.; Kouwenhoven, L. P.; Zwiller, V.; Borgström, M. T.; Wunnicke, O.; Verheijen, M. A.; Bakkers, E. P. A. M. Single Quantum Dot Nanowire LEDs. Nano Lett. 2007, 7, 367371.

(7) Heiss, M.; Fontana, Y.; Gustafsson, A.; Wust, G.; Magen, C.; O’Regan, D. D.; Luo, J. W.; Ketterer, B.; Conesa-Boj, S.; Kuhlmann, A. 
V.; Houel, J.; Russo-Averchi, E.; Morante, J. R.; Cantoni, M.; Marzari, N.; Arbiol, J.; Zunger, A.; Warburton, R. J.; Fontcuberta i Morral, A. Self-assembled Quantum Dots in a Nanowire System for Quantum Photonics. Nat. Mater. 2013, 12, 439-444.

(8) Peng, K.; Parkinson, P.; Fu, L.; Gao, Q.; Jiang, N.; Guo, Y.; Wang, F.; Joyce, H. J.; Boland, J. L.; Tan, H. H.; Jagadish, C.; Johnston, M. B. Single Nanowire Photoconductive Terahertz Detectors. Nano Lett. 2015, 15, 206-210.

(9) Vitiello, M. S.; Coquillat, D.; Viti, L.; Ercolani, D.; Teppe, F.; Pitanti, A.; Beltram, F.; Sorba, L.; Knap, W.; Tredicucci, A. RoomTemperature Terahertz Detectors Based on Semiconductor Nanowire Field-Effect Transistors. Nano Lett. 2012, 12, 96-101.

(10) Seletskiy, D. V.; Hasselbeck, M. P.; Cederberg, J. G.; Katzenmeyer, A.; Toimil-Molares, M. E.; Leonard, F.; Talin, A. A.; Sheik-Bahae, M. Efficient Terahertz Emission from InAs Nanowires. Phys. Rev. B: Condens. Matter Mater. Phys. 2011, 84, 115421.

(11) Czaban, J. A.; Thompson, D. A.; Lapierre, R. R. GaAs CoreShell Nanowires for Photovoltaic Applications. Nano Lett. 2009, 9, $148-154$.

(12) Tian, B.; Kempa, T. J.; Lieber, C. M. Single Nanowire Photovoltaics. Chem. Soc. Rev. 2009, 38, 16-24.

(13) Law, M.; Greene, L. E.; Johnson, J. C.; Saykally, R.; Yang, P. D. Nat. Mater. 2005, 4, 455-459.

(14) Joyce, H. J.; Docherty, C. J.; Gao, Q.; Tan, H. H.; Jagadish, C.; Lloyd-Hughes, J.; Herz, L. M.; Johnston, M. B. Electronic Properties Of GaAs, InAs and InP Nanowires Studied by Terahertz Spectroscopy. Nanotechnology 2013, 24, 214006.

(15) Titova, L. V.; Hoang, T. B.; Jackson, H. E.; Smith, L. M.; Yarrison-Rice, J. M.; Kim, Y.; Joyce, H. J.; Tan, H. H.; Jagadish, C. Temperature Dependence of Photoluminescence from Single Coreshell GaAs-AlGaAs Nanowires. Appl. Phys. Lett. 2006, 89, 173126.

(16) Tambe, M. J.; Lim, S. K.; Smith, M. J.; Allard, L. F.; Gradečak, S. Realization of Defect-Free Epitaxial Core-shell GaAs/AlGaAs Nanowire Heterostructures. Appl. Phys. Lett. 2008, 93, 151917.

(17) Breuer, S.; Pfüller, C.; Flissikowski, T.; Brandt, O.; Grahn, H. T.; Geelhaar, L.; Riechert, H. Suitability of Au- and Self-Assisted GaAs Nanowires for Optoelectronic Applications. Nano Lett. 2011, 11, 1276-1279.

(18) Demichel, O.; Heiß, M.; Bleuse, J.; Mariette, H.; Fontcuberta i Morral, A. Impact of Surfaces on the Optical Properties of GaAs Nanowires. Appl. Phys. Lett. 2010, 97, 201907.

(19) Smith, L. M.; Wolford, D. J.; Martinsen, J.; Venkatasubramanian, R.; Ghandhi, S. K. Photoexcited Carrier Lifetimes and Spatial Transport in Surface-Free GaAs Homostructures. J. Vac. Sci. Technol., B: Microelectron. Process. Phenom. 1990, 8, 787-792.

(20) Smith, L. M.; Wolford, D. J.; Venkatasubramanian, R; Ghandhi, S. K. Radiative Recombination in Surface-Free $n+/ n-/ n+$ GaAs Homostructures. Appl. Phys. Lett. 1990, 57, 1572-1574.

(21) Wolford, D. J.; Gilliland, G. D.; Kuech, T. F.; Smith, L. M.; Martinsen, J.; Bradley, J. A.; Tsang, C. F.; Venkatasubramanian, R.; Ghandi, S. K.; Hjalmarson, H. P. Intrinsic Recombination and Interface Characterization in Surface-Free GaAs Structures. J. Vac. Sci. Technol., B: Microelectron. Process. Phenom. 1991, 9, 2369-2376.

(22) Haraguchi, K.; Katsuyama, T.; Hiruma, K.; Ogawa, K. GaAs p-njunction Formed in Quantum Wire Crystals. Appl. Phys. Lett. 1992, 60, 745-747.

(23) Cui, Y.; Duan, X. F.; Hu, J. T.; Lieber, C. M. Doping and Electrical Transport in Silicon Nanowires. J. Phys. Chem. B 2000, 104, 5213-5216.

(24) Hilse, M.; Ramsteiner, M.; Breuer, S.; Geelhaar, L.; Riechert, H. Incorporation of the Dopants $\mathrm{Si}$ and Be into GaAs Nanowires. Appl. Phys. Lett. 2010, 96, 193104.

(25) Casadei, A.; Krogstrup, P.; Heiß, M.; Rohr, J. A.; Colombo, C.; Ruelle, T.; Upadhyay, S.; Sorensen, C. B.; Nygård, J.; Fontcuberta i Morral, A. Doping Incorporation Paths in Catalyst-Free Be-Doped GaAs Nanowires. Appl. Phys. Lett. 2013, 102, 013117.

(26) Dufouleur, J.; Colombo, C.; Garma, T.; Ketterer, B.; Uccelli, E.; Nicotra, M.; Fontcuberta i Morral, A. P-Doping Mechanisms in
Catalyst-Free Gallium Arsenide Nanowires. Nano Lett. 2010, 10, 1734-1740.

(27) Ketterer, B.; Mikheev, E.; Uccelli, E.; Fontcuberta i Morral, A. Compensation Mechanism in Silicon-Doped Gallium Arsenide Nanowires. Appl. Phys. Lett. 2010, 97, 223103.

(28) Xu, L. A.; Su, Y.; Chen, Y. Q.; Xiao, H. H.; Zhu, L. A.; Zhou, Q. T.; Li, S. Synthesis and Characterization of Indium-Doped $\mathrm{ZnO}$ Nanowires with Periodical Single-Twin Structures. J. Phys. Chem. B 2006, 110, 6637-6642.

(29) Algra, R. E.; Verheijen, M. A.; Borgström, M. T.; Feiner, L. F.; Immink, G.; Van Enckevort, W. J. P.; Vlieg, E.; Bakkers, E. P. A. M. Twinning Superlattices in Indium Phosphide Nanowires. Nature 2008, 456, 369-372.

(30) Dimakis, E.; Ramsteiner, M.; Tahraoui, A.; Riechert, H.; Geelhaar, L. Shell-Doping of GaAs Nanowires with Si for N-Type Conductivity. Nano Res. 2012, 5, 796-804.

(31) Suomalainen, S.; Hakkarainen, T. V.; Salminen, T.; Koskinen, R.; Honkanen, M.; Luna, E.; Guina, M. Te-Doping of Self-Catalyzed GaAs Nanowires. Appl. Phys. Lett. 2015, 107, 012101.

(32) Gutsche, C.; Lysov, A.; Regolin, I.; Blekker, K.; Prost, W.; Tegude, F. J. N-Type Doping of Vapor-Liquid-Solid Grown GaAs Nanowires. Nanoscale Res. Lett. 2011, 6, 65.

(33) Jadczak, J.; Plochocka, P.; Mitioglu, A.; Breslavetz, I.; Royo, M.; Bertoni, A.; Goldoni, G.; Smolenski, T.; Kossacki, P.; Kretinin, A.; Shtrikman, H.; Maude, D. K. Unintentional High-Density p-Type Modulation Doping of a GaAs/AlAs Core-Multishell Nanowire. Nano Lett. 2014, 14, 2807-2814.

(34) Sladek, K.; Klinger, V.; Wensorra, J.; Akabori, M.; Hardtdegen, H.; Grützmacher, D. MOVPE of N-Doped GaAs and Modulation Doped GaAs/AlGaAs Nanowires. J. Cryst. Growth 2010, 312, 635640.

(35) Spirkoska, D.; Fontcuberta i Morral, A.; Dufouleur, J.; Xie, Q.; Abstreiter, G. Free Standing Modulation Doped Core-Shell GaAs/ AlGaAs Hetero-nanowires. Phys. Status Solidi RRL 2011, 5, 353-355.

(36) Zervos, M. Delta $(\delta)$-Doping of Semiconductor Nanowires. Phys. Status Solidi RRL 2013, 7, 651-654.

(37) Cui, Z. X.; Perumal, R.; Ishikura, T.; Konishi, K.; Yoh, K.; Motohisa, J. Characterizing the Electron Transport Properties of a Single $\langle 110\rangle$ InAs Nanowire. Appl. Phys. Express 2014, 7, 085001.

(38) Prechtel, L.; Padilla, M.; Erhard, N.; Karl, H.; Abstreiter, G.; Fontcuberta i Morral, A.; Holleitner, A. W. Time-Resolved Photoinduced Thermoelectric and Transport Currents in GaAs Nanowires. Nano Lett. 2012, 12, 2337-2341.

(39) Boland, J. L.; Conesa-Boj, S.; Parkinson, P.; Tütüncüoglu; Matteini, F.; Rüffer; Casadei, A.; Amaduzzi, F.; Jabeen, F.; Davies, C. L.; Joyce, H. J.; Herz, L. M.; Fontcuberta i Morral, A.; Johnston, M. B. Modulation Doping of GaAs/AlGaAs Core-Shell Nanowires with Effective Defect Passivation and High Electron Mobility. Nano Lett. 2015, 15, 1336-1342.

(40) Storm, K.; Halvardsson, F.; Heurlin, M.; Lindgren, D.; Gustafsson, A.; Wu, P. M.; Monemar, B.; Samuelson, L. Spatially Resolved Hall Effect Measurement in a Single Semiconductor Nanowire. Nat. Nanotechnol. 2012, 7, 718-722.

(41) Chang, C.; Chi, C.; Yao, M.; Huang, N.; Chen, C.; Theiss, J.; Bushmaker, A. W.; LaLumondiere, S.; Yeh, T.; Povinelli, M. L.; Zhou, C.; Dapkus, P. D.; Cronin, S. B. Electrical and Optical Characterization of Surface Passivation in GaAs Nanowires. Nano Lett. 2012, 12, 44844489.

(42) Funk, S.; Royo, M.; Zardo, I.; Rudolph, D.; Morkötter, S.; Mayer, B.; Becker, J.; Bechtold, A.; Matich, S.; Döblinger, M.; Bichler, M.; Koblmüller, G.; Finlay, J. J.; Bertoni, A.; Goldoni, G.; Abstreiter, A. High Mobility One- and Two-Dimensional Electron Systems in Nanowire-Based Quantum Heterostructures. Nano Lett. 2013, 13, 6189-6196.

(43) Ketterer, B.; Uccelli, E.; Fontcuberta i Morral, A. Mobility and Carrier Density in p-Type GaAs Nanowires Measured by Transmission Raman Spectroscopy. Nanoscale 2012, 4, 1789-1793. 
(44) Parkinson, P.; Lloyd-Hughes, J.; Gao, Q.; Tan, H. H.; Jagadish, C.; Johnston, M. B.; Herz, L. M. Transient Terahertz Conductivity of GaAs Nanowires. Nano Lett. 2007, 7, 2162-2165.

(45) Parkinson, P.; Joyce, H. J.; Gao, Q.; Tan, H. H.; Zhang, X.; Zou, J.; Jagadish, C.; Herz, L. M.; Johnston, M. B. Carrier Lifetime and Mobility Enhancement in Nearly Defect-Free Core-Shell Nanowires Measured using Time-Resolved Terahertz Spectroscopy. Nano Lett. 2009, 9, 3349-3353.

(46) Ponseca, C. S.; Němec, H.; Wallentin, J.; Anttu, N.; Beech, J. P.; Iqbal, A.; M, B.; Pistol, M. E.; Samuelson, L.; Yartsev, A. Bulk-Like Transverse Electron Mobility in an Array of Heavily n-Doped InP Nanowires Probed by Terahertz Spectroscopy. Phys. Rev. B: Condens. Matter Mater. Phys. 2014, 90, 085405.

(47) Joyce, H. J.; Gao, Q.; Tan, H. H.; Jagadish, C.; Kim, Y.; Zou, J.; Smith, L. M.; Jackson, H. E.; Yarrison-Rice, J. M.; Parkinson, P.; Johnston, M. B. III-V Semiconductor Nanowires for Optoelectronic Device Applications. Prog. Quantum Electron. 2011, 35, 23-75.

(48) Maharjan, A.; Pemasiri, K.; Kumar, P.; Wade, A.; Smith, L. M.; Jackson, H. E.; Yarrison-rice, J. M.; Kogan, A.; Paiman, S.; Gao, Q.; Tan, H. H.; Jagadish, C. Room Temperature Photocurrent Spectroscopy of Single Zincblende and Wurtzite InP Nanowires. Appl. Phys. Lett. 2009, 94, 193115.

(49) Uccelli, E.; Arbiol, J.; Magen, C.; Krogstrup, P.; Russo-Averchi, E.; Heiß, M.; Mugny, G.; Morier-Genoud, F.; Nygård, J.; Morante, J. R.; Fontcuberta i Morral, A. Three-Dimensional Multiple-Order Twinning of Self-Catalyzed GaAs Nanowires on Si Substrates. Nano Lett. 2011, 11, 3827-3832.

(50) Matteini, F.; Tütüncüoglu; Potts, H.; Jabeen, F.; Fontcuberta i Morral, A. Wetting of $\mathrm{Ga}$ on $\mathrm{SiO}_{x}$ and its Impact on GaAs Nanowire Growth. Cryst. Growth Des. 2015, 15, 3105-3109.

(51) Colombo, C.; Heiß, M.; Grätzel, M.; Fontcuberta i Morral, A. Gallium Arsenide p-i-n Radial Structures for Photovoltaic Applications. Appl. Phys. Lett. 2009, 94, 173108.

(52) Casadei, A.; Schwender, J.; Russo-Averchi, E.; Rüffer, D.; Heiß, M.; Alarco-llado, E.; Jabeen, F.; Ramezani, M.; Nielsch, K.; Fontcuberta i Morral, A. Electrical Transport in C-Doped GaAs Nanowires: Surface Effects. Phys. Status Solidi RRL 2013, 7, 890-893.

(53) Birner, S.; Zibold, T.; Andlauer, T.; Kubis, T.; Sabathil, M.; Trellakis, A.; Vogl, P. Nextnano: General Purpose 3-D Simulations. IEEE Trans. Electron Devices 2007, 54, 2137-2142.

(54) Ahtapodov, L.; Todorovic, J.; Olk, P.; Mjaland, T.; Slattnes, P.; Dheeraj, D. L.; Fimland, B. O.; Weman, H. A Story Told by a Single Nanowire: Optical Properties of Wurtzite GaAs. Nano Lett. 2012, 12, 6090-6095.

(55) Lloyd-Hughes, J.; Jeon, T.-I. A Review of the Terahertz Conductivity of Bulk and Nanomaterials. J. Infrared, Millimeter, Terahertz Waves 2012, 33, 871.

(56) Strauss, U.; Rühe, W. W.; Köler, K. Auger Recombination in Intrinsic GaAs. Appl. Phys. Lett. 1993, 62, 55-57.

(57) Nienhuys, H.-K.; Sundström, V. Influence of Plasmons on Terahertz Conductivity Measurements. Appl. Phys. Lett. 2005, 87, 012101.

(58) Joyce, H. J.; Wong-Leung, J.; Yong, C.; Docherty, C. J.; Paiman, S.; Gao, Q.; Tan, H. H.; Jagadish, C.; Lloyd-Hughes, J.; Herz, L. M.; Johnston, M. B. Ultra-Low Surface Recombination Velocity in InP Nanowires Probed by Terahertz Spectroscopy. Nano Lett. 2012, 12, 5325-5330.

(59) Parkinson, P.; Dodson, C.; Joyce, H. J.; Bertness, K. A.; Sanford, N. A.; Herz, L. M.; Johnston, M. B. Non-Contact Measurement of Charge Carrier Lifetime and Mobility in GaN Nanowires. Nano Lett. 2012, 12, 4600-4604.

(60) Joyce, H. J.; Parkinson, P.; Jiang, N.; Docherty, C. J.; Gao, Q.; Tan, H. H.; Jagadish, C.; Herz, L. M.; Johnston, M. B. Electron Mobilities Approaching Bulk Limits in "Surface-Free" GaAs Nanowires. Nano Lett. 2014, 14, 5989-5994.

(61) Yu, P. Y.; Cardona, M. Fundamentals of Semiconductors, 3rd ed.; Springer, 2003.
(62) Pfeiffer, L.; West, K. W.; Stormer, H. L.; Baldwin, K. W. Electron Mobilities Exceeding $10^{7} \mathrm{~cm}^{2} / \mathrm{Vs}$ in Modulation-doped GaAs. Appl. Phys. Lett. 1989, 55, 1888-1890.

(63) Parkinson, P.; Knappke, C. E. I.; Kamonsutthipaijit, N.; Sirithip, K.; Matichak, J. D.; Anderson, H. L.; Herz, L. M. Ultrafast Energy Transfer in Biomimetic Multistrand Nanorings. J. Am. Chem. Soc. 2014, 136, 8217-8220. 